\title{
Observations of sausage mode oscillations in a flaring loop
}

\author{
V.F. Melnikov ${ }^{1}$, V.E. Reznikova ${ }^{1}$, K. Shibasaki ${ }^{2}$ and V.M. \\ Nakariakov $^{3}$ \\ ${ }^{1}$ Radiophysical Research Institute (NIRFI), Nizhny Novgorod, 603950, Russia \\ email:meln@nirfi.sci-nnov.ru, reznik@nirfi.sci-nnov.ru \\ ${ }^{2}$ Nobeyama Radio Observatory, NAOJ, Minamimaki, Minamisaku, Nagano 384-1305, Japan, \\ email:shibasak@nro.nao.ac.jp \\ ${ }^{3}$ Physics Department, University of Warwick, Coventry, CV4 7AL, UK \\ email:valery@astro.warwick.ac.uk
}

\begin{abstract}
We provide an observational proof of the existence of the global sausage mode oscillations in a flaring loop studying a microwave burst with quasi-periodical pulsations observed with the Nobeyama Radioheliograph (NoRH).
\end{abstract}

In this paper we show one of the first spatially resolved observations (see Asai et al. 2001; Melnikov et al. 2002; Grechnev et al. 2003) of microwave pulsations in a coronal loop. The observations were carried out with the Nobeyama Radioheliograph (NoRH) operating at the microwave frequencies 17 and $34 \mathrm{GHz}$ with high spatial (10"-5") and temporal (0.1 s) resolution (Nakajima et al. 1994). Observations without spatial resolution could not allow to identify the specific oscillation mode because of too many free parameters. The identification of the oscillation mode should be based upon the determination of the observational period, the longitudinal and transverse sizes of the magnetic loop, and the spatial distribution of the oscillation amplitude along the loop.

\section{Properties of the oscillating flaring loop}

The solar flare of 12 th of January, 2000 was observed by NoRH at both frequencies. The flare had a pronounced loop-like structure well seen on Yohkoh/SXT images. Microwave and HXT images agree well with this structure. Melnikov et al. 2002 found that the time profiles of the microwave emission at 17 and $34 \mathrm{GHz}$ exhibit synchronous quasiperiodical variations of the intensity in different parts of the corresponding flaring loop. The length of the flaring loop is estimated as $L=25 \mathrm{Mm}$ and its width at half intensity at $34 \mathrm{GHz}$ as about $2 a=6 \mathrm{Mm}$. The loop is estimated to be filled by a dense plasma with the concentration $n_{0} \approx 10^{17} \mathrm{~m}^{-3}$ penetrated by the magnetic field of the strength $B_{0} \approx$ $50-100 \mathrm{G}$. The pulsations of microwave emission flux from three boxes $(10$ " $\times 10$ ") located at the loop top and at its legs, and corresponding Fourier spectra of the pulsations are shown in Figure 1. There are two main spectral components of the observed pulsations, with periods $P_{1}=14-17 \mathrm{~s}$ and $P_{2}=8-11 \mathrm{~s}$, observed everywhere in the loop. However, their relative contribution depends on the location. The first one is more pronounced at the apex while the second one is relatively stronger at the loop legs. Detailed comparison of the curves in Fig. 1 a,b,c shows the pulsations at the legs to be almost synchronous with the quasi-period $P_{2}=8-11$ s. At the loop apex the synchronism with the legs pulsations is not so obvious but definitely exists on the larger time scale, $P_{1}=14-17 \mathrm{~s}$. 

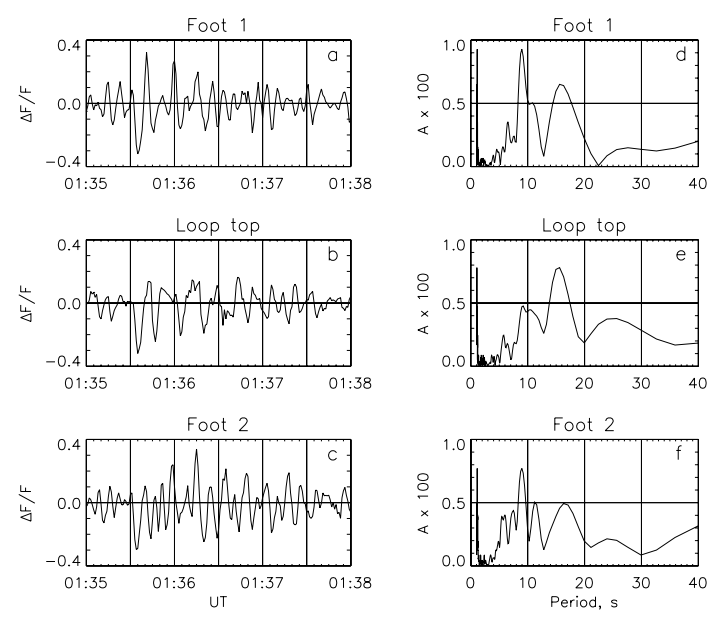

Figure 1. Left column: Radio flux variations $\Delta F / F=\left[F(t)-F_{0}\right] / F_{0}$, normalized on the slowly varying component of the emission, $F_{0}$, obtained by smoothing the observed flux $F(t)$ over $10 \mathrm{~s}$. Right column: The corresponding Fourier power spectra of the pulsations shown in figures a,b,c. Two dominant spectral components with periods $P_{1}=14-17 \mathrm{~s}$ and $P_{2}=8-11 \mathrm{~s}$ are clearly seen for pulsations situated at the different parts of the loop.

\section{Interpretation}

These properties of pulsations indicate the possibility of the simultaneous existence of two sausage modes of oscillations in the loop: the global one, with the period $P_{1}=14-17 \mathrm{~s}$ and the nodes at the footpoints, and a harmonic, with $P_{2}=8-11 \mathrm{~s}$. The global sausage mode has a maximum of the magnetic field perturbation at the loop apex and nodes at the footpoints. As it was demonstrated by Nakariakov et al. 2003 the existence condition for this mode is the ratio of the loop length to its diameter to be less than about a half of the square root of the density contrast ratio. We estimated the Alfvén speeds inside and outside the loop as $C_{A 0}=4.8 \times 10^{2} \mathrm{~km} / \mathrm{s}$ and $C_{A e}=(4.5-5.3) \times 10^{3} \mathrm{~km} / \mathrm{s}$, respectively. Thus, in the loop studied, the longest theoretically possible wavelength $\lambda$ of the trapped sausage mode is about $(22-25) a$. As the observed loop radius is about $1 / 8$ of its length, this loop can support the global sausage mode.

We exclude the interpretation of the observed periodicity in terms of another, kink, global mode because the value of the phase speed of this mode lies between $C_{A 0}$ and the kink speed $C_{k}$, which for the given parameters of the loop is $665 \mathrm{~km} / \mathrm{s}$, giving the period greater than $75 \mathrm{~s}$, which is very far from the observed value.

\section{Acknowledgements}

The work was partly supported by the RFBR grants No.02-02-39005, 04-02-16753 and by the Royal Society British-Russian Research Collaboration grant.

\section{References}

Asai A., Shimojo M., Isobe H., Morimoto T., Yokoyama T., Shibasaki K., \& Nakajima H. 2001 ApJ 562, L103.

Grechnev V.V., White S.M., Kundu M.R. 2003 ApJ 588, 1163.

Melnikov V.F, Reznikova V.E., \& Shibasaki K. 2002 Proc. of Intern. Conf. "Active processes on the Sun and stars", Eds. Zaitsev V.V. and Yasnov L.V., 225.

Nakajima H. et al. 1994 The Nobeyama Radioheliograph. Proc. of the IEEE., 82, 705.

Nakariakov V.M., Melnikov V.F., Reznikova V.E. 2003 A $\& A$ 412, L7. 